\title{
Long-term changes in the refractive effect of a toric intraocular lens on astigmatism correction
}

\author{
Ken Hayashi ${ }^{1} \cdot$ Motoaki Yoshida $^{1} \cdot$ Shunsuke Hayashi $^{2,3} \cdot$ Akira Hirata $^{1}$
}

Received: 22 April 2021 / Revised: 16 August 2021 / Accepted: 30 August 2021 / Published online: 8 September 2021

(c) The Author(s) 2021

\begin{abstract}
Purpose To examine the long-term changes in the astigmatism-correcting effect of a toric intraocular lens (IOL) after stabilization of surgically induced astigmatic changes due to cataract surgery.

Methods Unilateral eyes of 120 patients that received a toric IOL for against-the-rule (ATR) or with-the-rule (WTR) astigmatism were enrolled. Manifest refractive and anterior corneal astigmatism, and ocular residual astigmatism which is mainly derived from internal optics were examined preoperatively, at approximately 2 months postoperatively (baseline) and at $5 \sim 10$ years postbaseline. The astigmatism was decomposed to vertical/horizontal (Rx) and oblique components (Ry), which was compared between baseline and $5 \sim 10$ years postbaseline. Results In the eyes having ATR astigmatism, the mean Rx and Ry of the manifest refractive and corneal astigmatism significantly changed toward ATR astigmatism between the baseline and 5 10 years postbaseline $(p \leq 0.0304)$, but those of ocular residual astigmatism did not change significantly between the 2 time points. In the eyes having WTR astigmatism, the Rx and Ry of refractive, corneal, and ocular residual astigmatism did not change significantly between the 2 time points. Double-angle plots revealed an ATR shift in refractive and corneal astigmatism and no marked change in the ocular residual astigmatism in the eyes with ATR astigmatism, and there is no change in this astigmatism in the eyes with WTR astigmatism. Conclusion The long-term changes with age in the effect of a toric IOL significantly deteriorated due to an ATR shift of corneal astigmatism in the eyes having ATR astigmatism, while it was maintained in eyes having WTR astigmatism, suggesting that ATR astigmatism should be overcorrected.
\end{abstract}

Keywords Cataract surgery $\cdot$ Long-term astigmatic changes with age $\cdot$ Toric intraocular lens $\cdot$ Against-the-rule astigmatism $\cdot$ With-the-rule astigmatism

\section{Key messages}

- Previous studies report that the visual effects and rotational stability of toric IOLs are well maintained over 1- or 2-year follow-up periods, but the long-term changes in the effects of toric IOLs was not examined for postoperative periods of longer than 2 years.

- The long-term effect of a toric IOL on correcting manifest refractive astigmatism significantly deteriorated due to an ATR shift of corneal astigmatism with age in eyes having ATR astigmatism during $5 \sim 10$ years postoperatively, while it was maintained in eyes having WTR astigmatism.

- The ocular residual astigmatism, which mainly consists of the effect of a toric IOL, and uncorrected and corrected distance visual acuity did not change significantly for $5 \sim 10$ years postoperatively.

Ken Hayashi

hayashi-ken@ hayashi.or.jp

Extended author information available on the last page of the article 


\section{Introduction}

Toric intraocular lenses (IOLs) effectively correct preexisting corneal astigmatism when implanted during cataract surgery and substantially improve uncorrected visual acuity [1-5]. Many novel devices have been developed for precisely aligning the meridian of toric IOLs, including image-guided system and intraoperative aberrometers, resulting in improvements in short-term visual outcomes [6-9]. Additionally, multifocal IOLs with a toric component can enhance the multifocal effect in eyes with excessive preexisting corneal astigmatism [10-13].

The long-term changes in the visual effects of toric IOLs on astigmatic correction remain unclear. Some studies report that the visual effects and rotational stability of toric IOLs are well maintained over 1- or 2-year follow-up times [14-18]. For example, Miyake et al. [16] reported excellent visual outcomes and rotational stability for up to 2 years after surgery. The long-term changes in the effects of toric IOLs for astigmatism correction after stabilization of surgically induced astigmatic change, however, have not been evaluated for longer postoperative intervals.

The present study examined the changes with age in the astigmatism-correcting effects of toric IOLs for $5 \sim 10$ years after stabilization of surgically induced astigmatic changes. Because astigmatism in the posterior cornea is an againstthe-rule (ATR) astigmatism in most eyes and has a nonnegligible effect in determining the IOL power $[19,20]$, the refractive effects may differ between eyes having ATR astigmatism and eyes having with-the-rule (WTR) astigmatism when the toric IOL cylindrical power and model are selected on the basis of data from only the anterior cornea [21-23]. Accordingly, in the present study, the long-term changes in the effects of a toric IOL were separately evaluated in eyes having preoperative ATR or WTR astigmatism.

\section{Patients and methods}

\section{Study design}

This study was a retrospective observational study conducted at the Hayashi Eye Hospital, Fukuoka, Japan, between February 26 and October 14, 2020. The study protocol was approved by the Institutional Review Board of the Hayashi Eye Hospital on February 25, 2020. The study protocol adhered to the tenets of the Declaration of Helsinki.

\section{Participants}

The medical records of all patients who had undergone cataract surgery with a toric IOL implantation performed by a single surgeon (KH) between 2010 and 2015 were reviewed beginning on February 26, 2020. The eyes that were continuously followed up at the Hayashi Eye Hospital for $5 \sim 10$ years after surgery were included. Inclusion criteria were (1) eyes that underwent phacoemulsification using an approximately $2.4-\mathrm{mm}$ clear corneal incision; (2) eyes that received a 1-piece hydrophobic acrylic IOL with a toric component (SA60AT or SN60AT; Alcon Laboratories, Fort Worth, Texas, USA); (3) eyes that were examined at least twice using an autokerato/refractometer at approximately 2 months postoperatively, and a difference in corneal astigmatism between the 2 examinations was within $0.5 \mathrm{D}$ in cylindrical power and within $\pm 15^{\circ}$ in the cylindrical axis (the latter examination was defined as baseline); (4) eyes that underwent examinations using an autokerato/refractometer at $5 \sim 10$ years after the baseline measurement; (5) first-operated eye when the patient underwent cataract surgery in both eyes; (6) eyes that underwent uneventful cataract surgery with no sutures; (7) eyes with no severe pathology of the cornea, optic nerve, or macula; and (8) eyes without previous history of other surgery or inflammation. Patient enrollment was continued until 120 eyes (60 eyes in each of the ATR and WTR astigmatism groups) were enrolled in the study, with the last eye enrolled on July 30, 2020.

\section{IOL power calculation and toric meridian determination}

The spherical power of the IOL was calculated using the SRK/T formula with the optimized A-constant. For IOL power determination, the axial length was measured using the IOLMaster (Carl Zeiss Meditec GmbH, Jena, Germany). In the eyes with a dense cataract, the axial length was measured using an ultrasonic applanation biometer (Ocuscan $^{\mathrm{TM}}$ Alcon Biophysic, Ferrand, France). The corneal curvature at the steepest and flattest meridians of the anterior cornea was measured by anterior segment optical coherence tomography (CASIA 1; TOMEY, Tokyo Japan), and the mean value of both meridians was used. The appropriate model and meridian of the toric IOL to achieve emmetropia was determined using an online toric IOL calculator program (available at http://www.acrys oftoriccalculator.com; Alcon). Preoperative keratometry and biometry data, incision location and size, and the surgeon's estimated surgically induced corneal astigmatism were entered into this calculator program.

The appropriate meridian of the toric IOL was determined utilizing the CASIA 1 dataset. Prominent structures of the iris or conjunctival vessels were selected as reference points on the screen of the CASIA 1, and the meridian of the toric IOL was indicated by the clockwise 
or counterclockwise angle from the reference structures. The angles from the reference points were used to place the toric IOL at the appropriate meridian.

\section{Surgical procedures}

A single surgeon $(\mathrm{KH})$ performed all cataract surgeries [13]. Just before surgery, with the patient seated upright at the slit-lamp to compensate for cyclorotation, the surgeon marked the corneal limbus at $0^{\circ}, 90^{\circ}, 180^{\circ}$, and $270^{\circ}$ using a marker after vertical alignment of the patient's head. At the beginning of the surgery, the steepest meridian of the corneal limbus was identified and marked by a steel knife using a toric IOL marker (9-705R1; Duckworth \& Kent, Hertfordshire, UK) according to the pre-marked points at the corneal limbus and the predetermined reference points on the iris or conjunctival structures. The surgeon made an approximately $2.4-\mathrm{mm}$ clear corneal incision horizontally for eyes having ATR corneal astigmatism, and superiorly for eyes having WTR astigmatism; the horizontal incision was made temporally in the right eye and nasally in the left eye. Phacoemulsification surgery was performed according to the previously described procedure [13]. First, a continuous curvilinear capsulorrhexis measuring approximately $5.0 \mathrm{~mm}$ in diameter was made using a bent needle. After hydrodissection, phacoemulsification of the nucleus and aspiration of the residual cortex was conducted. Without enlarging the incision, the lens capsule was inflated with sodium hyaluronate 1\% (Healon; Johnson \& Johnson Vision Santa Ana, California, USA or Hyaguard; Nitten Pharmaceutical, Tokyo, Japan), after which the IOL was placed into the capsular bag. The toric IOL was rotated until the toric IOL indentations were aligned with the marked steepest meridian of corneal astigmatism. All viscoelastic materials were thoroughly aspirated, while carefully avoiding rotation of the IOL. IOL meridian alignment was confirmed after wound closure using stromal hydration. In this series, all surgeries were uneventful, and no sutures were required in any of the cases. measurements was repeated at least 4 times, and the mean value was used for analysis. The TONOREF I and II show a specific reliability index of each measurement ranging from 5 to 9 , with 9 being the most reliable. When the optic medium is not clear due to corneal edema, anterior chamber inflammation, or cataract, the reliability index decreases. Only measurement values with a high-reliability index of 8 and 9 were included in the analysis. Manifest refractive spherical equivalent was determined as the spherical power plus half the cylindrical power. The magnitude and meridian of the anterior corneal astigmatism were also measured using TONOREF I and II. Furthermore, ocular residual astigmatism which was defined as the difference between manifest refractive astigmatism and anterior corneal astigmatism by Alpins was calculated [24, 25].

Manifest refractive astigmatism was converted to the value at the corneal plane for calculating ocular residual astigmatism and changed back to the value at the spectacle plane for data presentation. Ocular residual astigmatism was assumed to comprise overall astigmatism mainly due to the toric IOL, as well as IOL tilt/ decentration, posterior corneal astigmatism, and other internal optic factors. Ocular residual astigmatism was calculated at the corneal plane. The type of astigmatism (WTR astigmatism, ATR astigmatism, or oblique astigmatism) was also recorded. Corneal astigmatism in which the steeper meridian was between $60^{\circ}$ and $120^{\circ}$ was defined as WTR astigmatism, that in which the steeper meridian was between $0^{\circ}$ and $30^{\circ}$ or between $150^{\circ}$ and $180^{\circ}$ was defined as ATR astigmatism and that in which the steeper meridian was between $30^{\circ}$ and $60^{\circ}$ or between $120^{\circ}$ and $150^{\circ}$ was defined as oblique astigmatism. Uncorrected or corrected distance visual acuity on decimal charts was recorded at all visits, and the decimal visual acuity was converted to the logarithm of minimal angle of resolution (logMAR) scale for statistical analysis.

Ocular residual astigmatism $=$ manifest refractive astigmatism - corneal astigmatism

\section{Outcome measures}

All patients underwent examinations preoperatively and at approximately 2 months postoperatively (baseline) and at 5 10 years after baseline. Manifest refractive spherical and cylindrical powers were measured objectively using an autokerato/refractometer (TONOREF I and II, NIDEK, Gamagori, Japan). To precisely measure the refractive states, automated capture of 3
Data on the astigmatism of the manifest refractive, anterior corneal, and ocular residual astigmatism at baseline and at $5 \sim 10$ years after baseline were used to analyze the astigmatic change over a period of at least 5 years. The astigmatism was decomposed to vertical $\left(90^{\circ}\right)$ /horizontal $\left(180^{\circ}\right)$ and oblique $\left(45^{\circ}\right.$ and $\left.135^{\circ}\right)$ astigmatism components using the $\mathrm{X}-\mathrm{Y}$ coordinate analysis, originally described by Naeser and Hjortdal [26]. This analysis defines the vertical/ horizontal astigmatism component as the Rx and oblique 
astigmatism components as the Ry. A negative Rx value indicates an ATR astigmatism, while a positive $\mathrm{Rx}$ indicates a WTR astigmatism. In the present study, the magnitude and meridian of manifest refractive, anterior corneal, and ocular residual astigmatism at baseline and at $5 \sim 10$ years postoperatively were plotted on double-angle plots utilizing the ASCRS Astigmatism Double Angle Plot Tool (available at http://ascrs.org/) [27]. The centroid values of astigmatism at baseline and at $5 \sim 10$ years after baseline were also revealed using this tool.

\section{Statistical analysis}

The normality of the data distribution was evaluated by inspecting histograms. The data for the manifest refractive, corneal, ocular residual astigmatism ( $\mathrm{Rx}$ and Ry values), manifest refractive spherical equivalent, uncorrected and corrected distance logMAR VA, and other continuous variables followed a normal distribution, and therefore, parametric analyses were used in this study. The univariate change in manifest refractive, anterior corneal, and ocular residual astigmatism ( $\mathrm{Rx}$ and Ry values) between the baseline and $5 \sim 10$ years after baseline were compared using a paired $t$ test. The bivariate changes in manifest refractive, corneal, and ocular residual astigmatism between the 2 time points were compared using a multivariate analysis of variance (MANOVA) [26]. Statistical power was calculated using the PASS 14 (NCSS LLC, Kaysville, Utah, USA). Differences in the age, preoperative manifest refractive spherical equivalent, preoperative and postoperative uncorrected and corrected distance logMAR VA, and other continuous variables between the ATR and WTR groups were compared using an unpaired $t$ test. The ratio of males to females, the ratio of left and right eyes, and other categorical variables between the ATR and WTR groups were compared using the chi-square test or Fisher's exact test where applicable. Any differences with a $p$ value less than 0.05 were considered statistically significant.

\section{Results}

All data of the 120 enrolled eyes were collected. Mean patient age ( \pm standard deviation) was $68.7 \pm 8.3$ years (range 43 to 84 years), and there were 55 men and 65 women. Preoperative patient characteristics and those at baseline are shown in Table 1. Mean age and the ratio of males to females were significantly higher in the ATR astigmatism group than in the WTR astigmatism group $(p<0.0001$ and $p=0.0032$, respectively). Preoperatively, the mean magnitude of the anterior corneal astigmatism and manifest refractive spherical equivalent differed significantly between the ATR and WTR astigmatism groups $(p \leq 0.0030)$. The mean time interval between the surgery and the baseline evaluation and between the baseline evaluation and that at $5 \sim 10$ years after baseline did not differ significantly between the ATR and WTR astigmatism groups. At baseline, the magnitude of corneal astigmatism and manifest refractive spherical equivalent did not differ significantly between the 2 groups.

Table 1 Comparison of patient characteristics before surgery and at baseline between eyes having against-the-rule (ATR) astigmatism and eyes having with-the-rule astigmatism (WTR)

\begin{tabular}{llll}
\hline Characteristics & ATR astigmatism $(n=60)$ & WTR astigmatism $(n=60)$ & $p$ value \\
\hline Preoperatively & & $64.35 \pm 8.09$ & $<0.0001^{*}$ \\
Age & $73.12 \pm 6.04$ & $19 \mathrm{M} / 41 \mathrm{~F}$ & $0.0032^{*}$ \\
Gender (male/female) & $36 \mathrm{M} / 24 \mathrm{~F}$ & $26 \mathrm{~L} / 34 \mathrm{R}$ & 0.4648 \\
Left/right & $31 \mathrm{~L} / 29 \mathrm{R}$ & $2.48 \pm 0.94$ & $0.0030^{*}$ \\
Corneal astigmatism (D) & $1.98 \pm 0.86$ & $-5.35 \pm 5.45(-20.25-1.75)$ & $<0.0001 *$ \\
MRSE (D) (range) & $-1.23 \pm 3.51(-16.75-2.13)$ & $0.48 \pm 0.31$ & 0.1828 \\
LogMAR CDVA & $0.41 \pm 0.20$ & & 0.4502 \\
Baseline & & $2.53 \pm 0.72$ & 0.4107 \\
Time interval between surgery and baseline (months) & $2.43 \pm 0.72$ & $6.83 \pm 1.67$ & 0.0605 \\
Time interval between baseline and 5 10 years after & $6.60 \pm 1.42$ & $-0.76 \pm 0.90$ & 0.3356 \\
baseline (years) & & $-0.76 \pm 0.90(-5.63-0.63)$ \\
Corneal astigmatism (D) & $1.77 \pm 0.81$ & $-0.62 \pm 0.66(-3.13-0.50)$ & \\
MRSE (D) (range) & &
\end{tabular}

$M$, male; $F$, female; $D$, diopter; MRSE, manifest refractive spherical equivalent; $C D V A$, corrected distance visual acuity; LogMAR, logarithm of minimal angle of resolution

* Significant difference between eyes having ATR astigmatism and WTR astigmatism 
Table 2 Univariate comparison of the mean $( \pm$ standard deviation) vertical/horizontal $(\mathrm{Rx})$ and oblique (Ry) components of manifest refractive, anterior corneal, and ocular residual astigmatism between the baseline and $5 \sim 10$ years after baseline in eyes having against-therule (ATR) astigmatism preoperatively

\begin{tabular}{|c|c|c|c|}
\hline Endpoint & Baseline & $5 \sim 10$ years after baseline & $p$ value \\
\hline \multicolumn{4}{|c|}{ Manifest refractive astigmatism (D) } \\
\hline Rx (range) & $-0.36 \pm 0.34(-1.24-0.71)$ & $-0.59 \pm 0.43(-1.95-0.12)$ & \multirow[t]{2}{*}{$0.0018^{*}$} \\
\hline Ry (range) & $0.04 \pm 0.37(-1.01-1.36)$ & $0.04 \pm 0.38(-1.38-1.43)$ & \\
\hline \multicolumn{4}{|c|}{ Anterior corneal astigmatism (D) } \\
\hline Rx (range) & $-0.76 \pm 0.39(-1.84-0.34)$ & $-0.99 \pm 0.53(-2.76$ to -0.38$)$ & $0.0081^{*}$ \\
\hline Ry (range) & $0.07 \pm 0.46(-1.92-1.14)$ & $0.09 \pm 0.51(-2.24-1.41)$ & 0.8953 \\
\hline \multicolumn{4}{|c|}{ Ocular residual astigmatism (D) } \\
\hline Rx (range) & $0.44 \pm 0.38(-0.49-1.31)$ & $0.46 \pm 0.48(-0.82-2.04)$ & 0.8723 \\
\hline Ry (range) & $-0.04 \pm 0.36(-1.03-0.91)$ & $-0.05 \pm 0.39(-1.36-0.86)$ & 0.8540 \\
\hline
\end{tabular}

\section{Univariate and bivariate comparisons of manifest refractive, corneal, and ocular residual astigmatism between the baseline and $5 \sim 10$ years after baseline}

Univariate comparisons using the paired $t$ test indicated that, in the eyes having ATR astigmatism preoperatively, the mean $\mathrm{Rx}$ value of manifest refractive astigmatism and corneal astigmatism significantly decreased toward a negative value from the baseline to $5 \sim 10$ years after baseline $(p \leq 0.0018)$, and the mean Ry value did not change significantly between the 2 time points (Table 2), indicating an ATR shift of manifest refractive and corneal astigmatism over a period of $5 \sim 10$ years. The mean Rx and Ry values of ocular residual astigmatism did not change significantly between the 2 time points. In the eyes with WTR astigmatism, the mean Rx and Ry values of manifest refractive, corneal, and ocular residual astigmatism did not change significantly between the baseline and 5 10 years after baseline (Table 3).

Bivariate comparisons using the MANOVA revealed that, in the eyes having ATR astigmatism preoperatively, the mean Rx and Ry values of manifest refractive astigmatism and anterior corneal astigmatism significantly changed toward ATR astigmatism from baseline to $5 \sim 10$ years after baseline $(p \leq 0.0304)$, and the mean $\mathrm{Rx}$ and Ry values of ocular residual astigmatism did not change significantly between the 2 time points (Fig. 1). In the eyes having WTR astigmatism preoperatively, the mean Rx and Ry values of manifest refractive, corneal, and ocular residual astigmatism did not change significantly between the 2 time points (Fig. 2). When we assumed an $\mathrm{Rx}$ value of $0.20 \mathrm{D}$ and an Ry value of $0.20 \mathrm{D}$ of manifest refractive astigmatism to be a clinically meaningful magnitude of difference between the 2 time points using the MANOVA, power analyses indicated that 60 patients provided a statistical power of $92.4 \%$ in eyes having ATR astigmatism and $93.3 \%$ in eyes having WTR astigmatism, indicating that the statistical power of the study was sufficient to detect a meaningful change in the main outcome measures.

\section{Changes in double-angle plots between the baseline and $5 \sim 10$ years after baseline}

The double-angle plots revealed that, in the eyes having ATR astigmatism preoperatively, the manifest
Table 3 Univariate comparison of the mean $( \pm$ standard deviation) vertical/horizontal (Rx) and oblique (Ry) components of manifest refractive, anterior corneal, and ocular residual astigmatism between the baseline and $5 \sim 10$ years after baseline in eyes having with-therule (WTR) astigmatism preoperatively

\begin{tabular}{|c|c|c|c|}
\hline Endpoint & Baseline & $5 \sim 10$ years after baseline & $p$ value \\
\hline \multicolumn{4}{|c|}{ Manifest refractive astigmatism (D) } \\
\hline Rx (range) & $0.00 \pm 0.44(-1.36-1.23)$ & $0.05 \pm 0.48(-1.36-1.35)$ & 0.5761 \\
\hline Ry (range) & $-0.10 \pm 0.30(-0.91-0.62)$ & $-0.07 \pm 0.32(-0.76-0.65)$ & 0.5539 \\
\hline \multicolumn{4}{|c|}{ Anterior corneal astigmatism (D) } \\
\hline Rx (range) & $0.94 \pm 0.57(-0.17-3.00)$ & $0.91 \pm 0.55(-0.40-2.37)$ & 0.7248 \\
\hline Ry (range) & $-0.12 \pm 0.39(-1.11-0.81)$ & $-0.06 \pm 0.43(-0.82-1.14)$ & 0.4460 \\
\hline \multicolumn{4}{|c|}{ Ocular residual astigmatism (D) } \\
\hline Rx (range) & $-0.95 \pm 0.45(-2.25-0.27)$ & $-0.86 \pm 0.43(-2.05-0.20)$ & 0.2624 \\
\hline Ry (range) & $0.03 \pm 0.35(-0.76-1.12)$ & $0.01 \pm 0.49(-1.11-1.12)$ & 0.8141 \\
\hline
\end{tabular}

$D$, diopters 
Fig. 1 Bivariate comparison of the mean ( \pm standard deviation) vertical/horizontal $(\mathrm{Rx})$ and oblique (Ry) components of the manifest refractive, anterior corneal, and ocular residual astigmatism between the baseline and $5 \sim 10$ years after baseline in eyes having against-the-rule astigmatism preoperatively

\section{Manifest refractive}

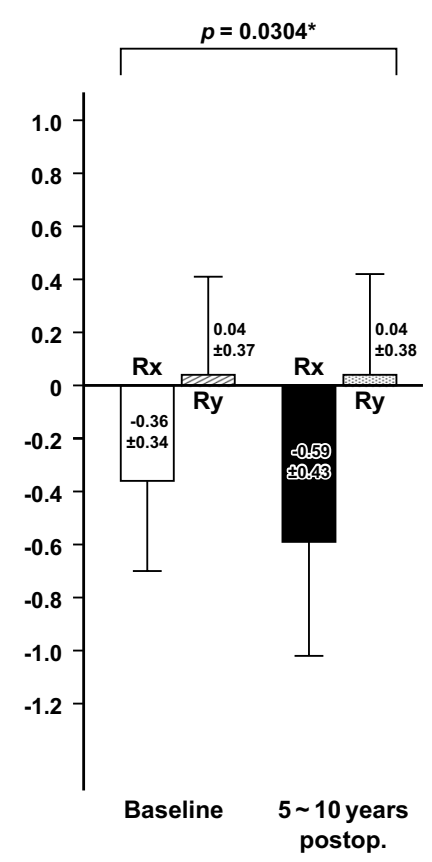

\section{Corneal}
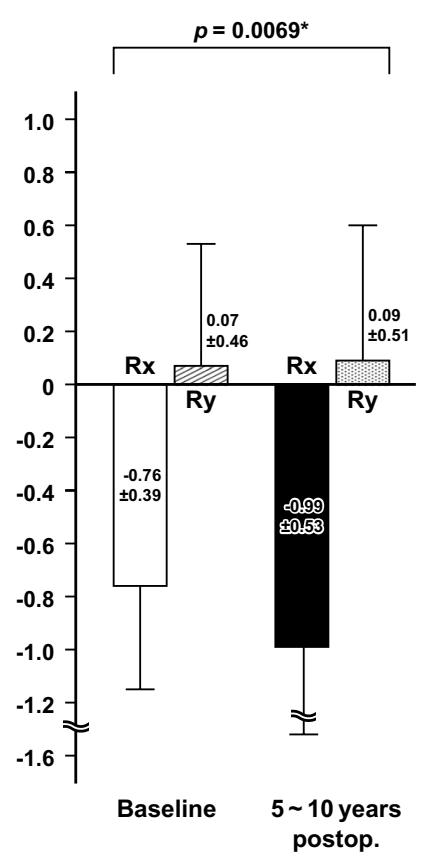

Ocular residual

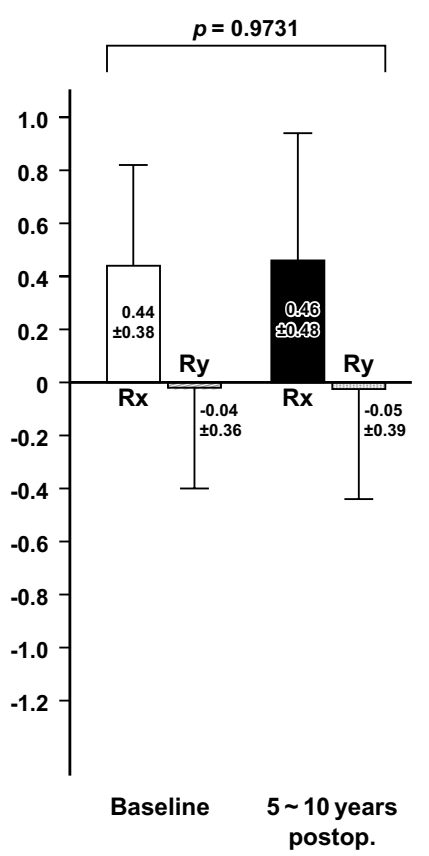

refractive astigmatism and anterior corneal astigmatism increased toward an ATR astigmatism from the baseline to $5 \sim 10$ years after baseline, and the ocular residual astigmatism did not change markedly (Fig. 3). In the eyes having the WTR astigmatism preoperatively, the manifest refractive, corneal, and ocular residual astigmatism did not change markedly between the 2 time points (Fig. 4).

\section{Comparison of uncorrected and corrected distance visual acuity between the baseline and $5 \sim 10$ years after baseline}

In eyes having either ATR and WTR astigmatism preoperatively, the mean uncorrected and corrected distance visual acuity did not worsen from the baseline to $5 \sim 10$ years after baseline (Table 4 ).

Fig. 2 Bivariate comparison of the mean ( \pm standard deviation) vertical/horizontal ( $\mathrm{Rx}$ ) and oblique (Ry) astigmatism components of the manifest refractive, anterior corneal, and ocular residual astigmatism between the baseline and 5 10 years after baseline in eyes having with-the-rule astigmatism preoperatively

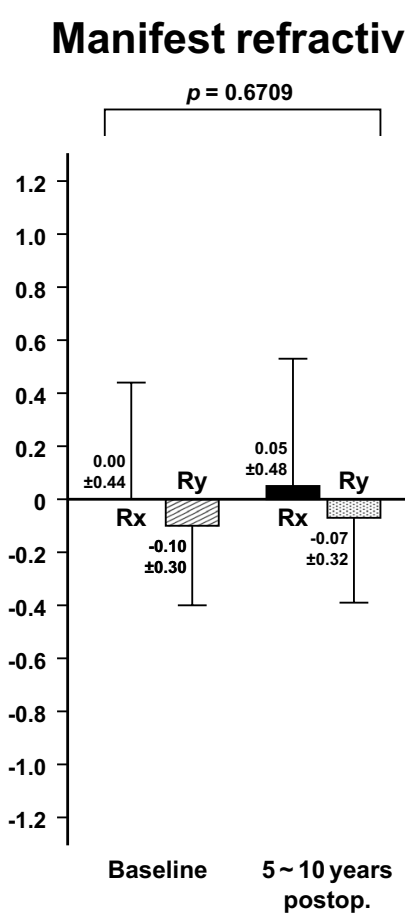

Corneal

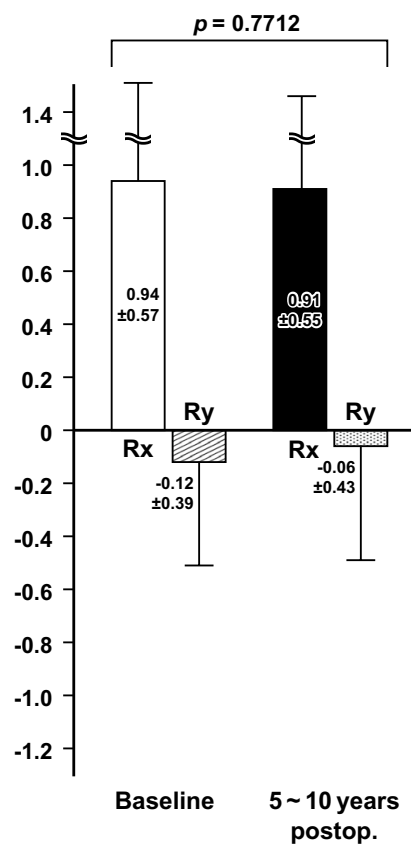

Ocular residual

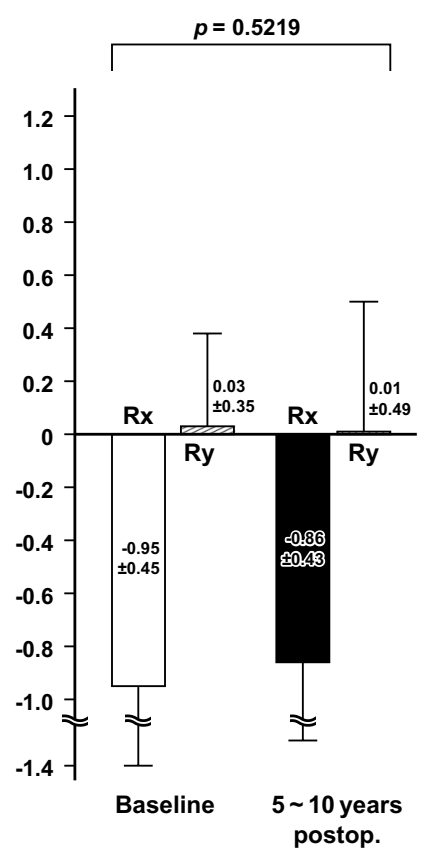




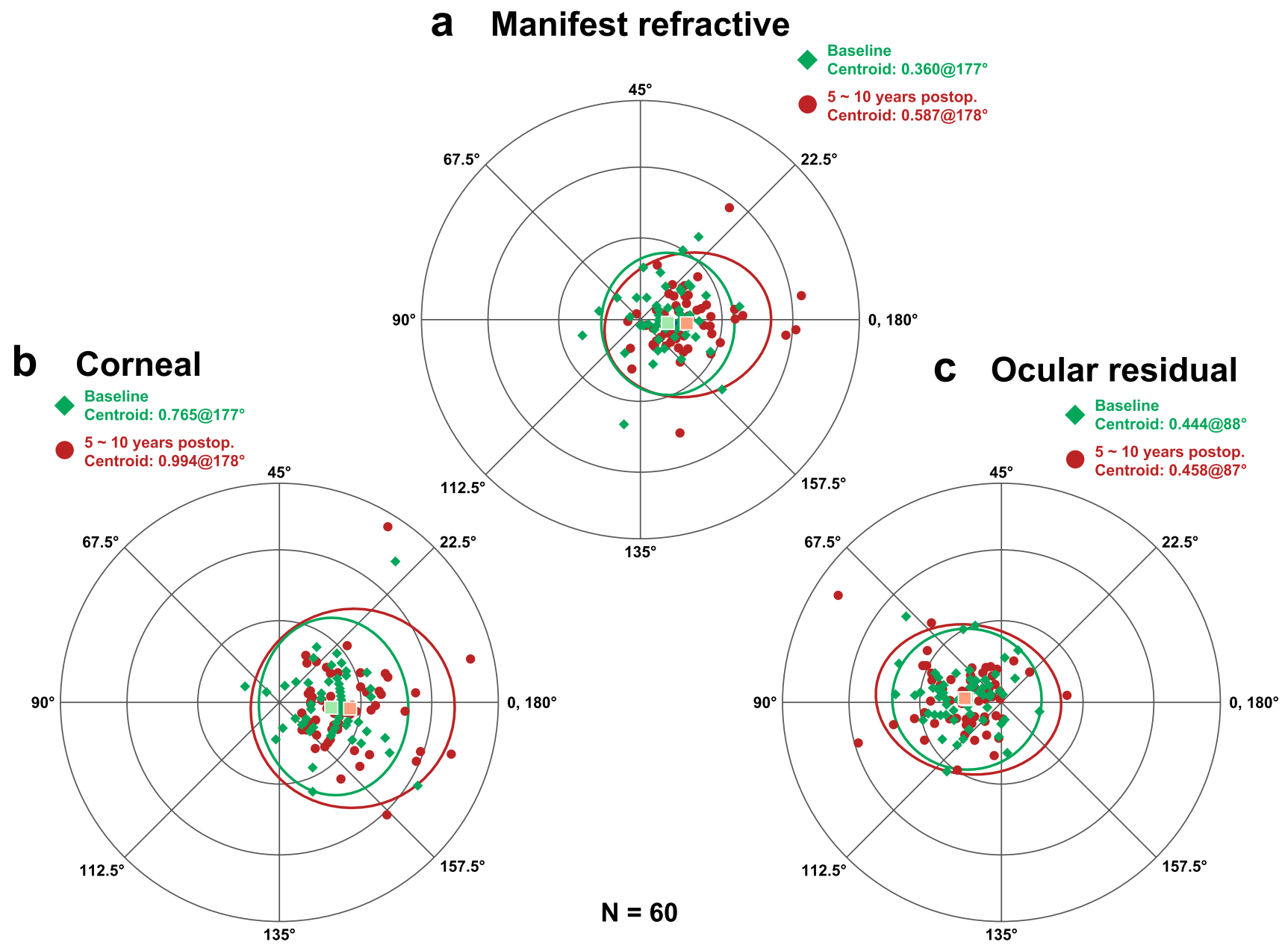

Fig. 3 Double-angle plot analysis of the manifest refractive (a), anterior corneal (b), and ocular residual astigmatism (c) between the baseline (green square) and 5 10 years after baseline (red circle) in eyes having against-the-rule astigmatism preoperatively

\section{Discussion}

The present study revealed that, in the eyes having ATR astigmatism before implantation of a toric IOL, the amount of ATR astigmatism of the manifest refractive astigmatism increased significantly in association with an ATR shift of corneal astigmatism over a period of $5 \sim 10$ years after stabilization of the astigmatic correcting effect of a toric IOL. The ocular residual astigmatism, which mainly consists of the effect of a toric IOL, did not differ significantly between the baseline and $5 \sim 10$ years after baseline. In the eyes having WTR astigmatism preoperatively, the amounts of manifest refractive, corneal, and ocular residual astigmatism did not worsen over the $5 \sim 10$ years after baseline. These findings suggest that the long-term effects of a toric IOL for correcting the manifest refractive astigmatism deteriorate due to the ATR shift of corneal astigmatism in eyes having ATR astigmatism, while they are maintained, at least over $5 \sim 10$ years postoperatively, in the eyes having WTR astigmatism.
Mean uncorrected distance visual acuity did not worsen between the baseline and 5 10 years after baseline in eyes having either ATR or WTR astigmatism. This finding indicates that the change in manifest refractive astigmatism over $5 \sim 10$ years postbaseline did not significantly affect uncorrected visual acuity. The mean follow-up duration, however, was 6.6 years in eyes having ATR astigmatism and 6.8 years in eyes having WTR astigmatism. Because an ATR shift in corneal and refractive astigmatism progresses with age [28-32], uncorrected distance visual acuity must deteriorate beyond 10 years. Furthermore, the mean change in manifest refractive astigmatism was $0.23 \mathrm{D}$ in eyes having ATR astigmatism and $0.05 \mathrm{D}$ in eyes having WTR astigmatism. Thus, because the difference in the astigmatic change between eyes having ATR and WTR astigmatism was small, it did not lead to a marked difference in impairment of uncorrected visual acuity.

Corneal astigmatism is mainly WTR astigmatism in younger individuals and continues to change toward ATR astigmatism with advancing age [28-31, 33]. On average, 


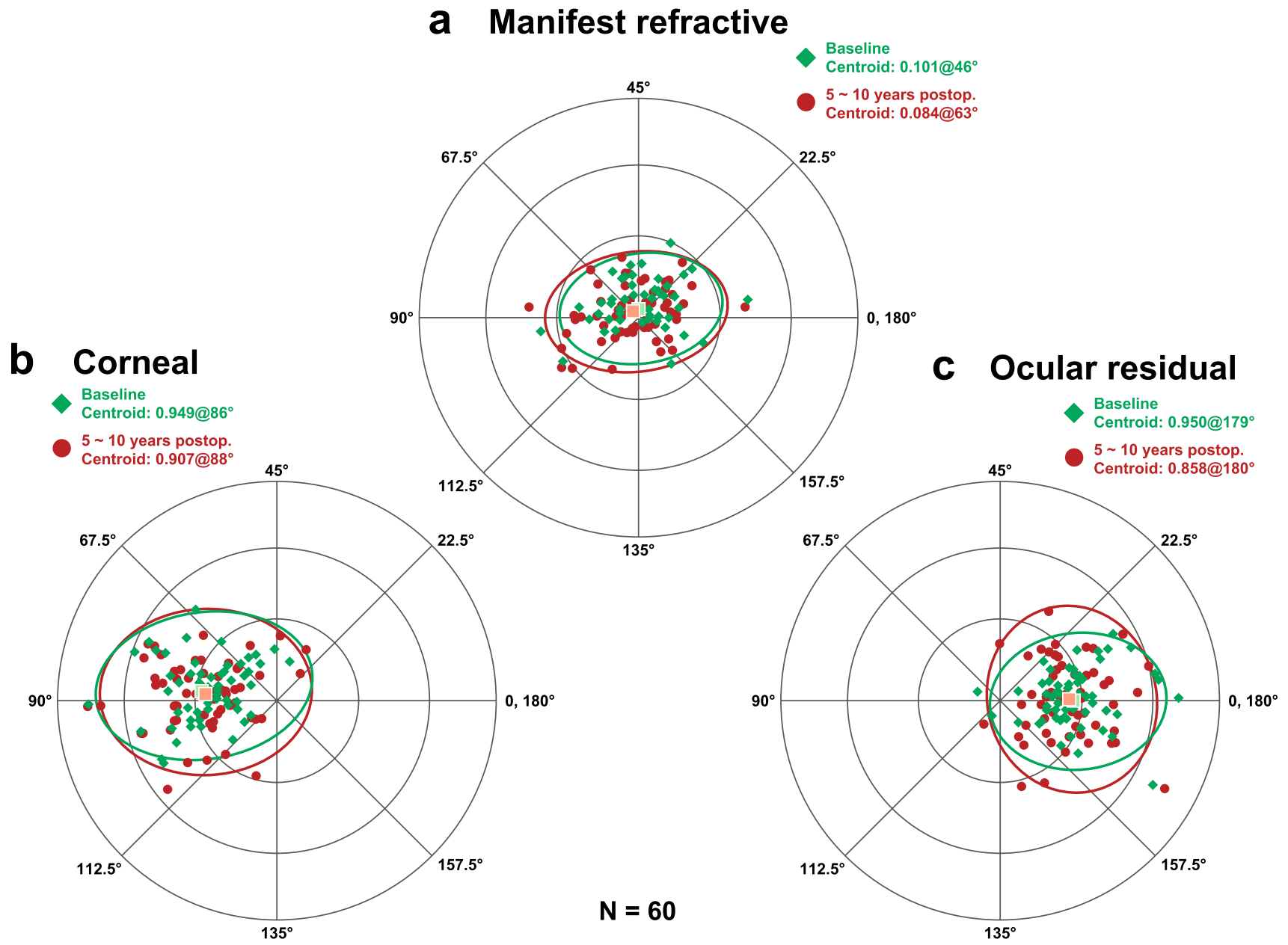

Fig. 4 Double-angle plot analysis of the manifest refractive (a), anterior corneal (b), and ocular residual astigmatism (c) between the baseline (green square) and 5 10 years after baseline (red circle) in eyes having with-the-rule astigmatism preoperatively

the type of corneal astigmatism changes from WTR astigmatism to ATR astigmatism at 60 years of age [28-31]. This age-related ATR change in corneal astigmatism also occurs to a similar extent in eyes that undergo cataract surgery $[28,29]$. In the present study, because the mean age of eyes having ATR astigmatism before surgery was

Table 4 Comparison of the mean ( \pm standard deviation) uncorrected and corrected distance logarithm of minimal angle of resolution (LogMAR) visual acuity between the baseline and the day at $5 \sim 10$ years after baseline

\begin{tabular}{lll}
\hline Endpoint $\quad$ Baseline & $\begin{array}{l}5 \sim 10 \text { years after } \\
\text { baseline }\end{array}$
\end{tabular}

Eyes having against-the-rule astigmatism preoperatively

$\begin{array}{llll}\text { Uncorrected } & 0.24 \pm 0.23 & 0.28 \pm 0.22 & 0.3466 \\ \text { Corrected } & 0.03 \pm 0.09 & 0.04 \pm 0.09 & 0.4489\end{array}$

Eyes having with-the-rule astigmatism preoperatively

\begin{tabular}{lrrr} 
Uncorrected & $0.18 \pm 0.21$ & $0.24 \pm 0.24$ & 0.1486 \\
Corrected & $-0.01 \pm 0.07$ & $0.00 \pm 0.07$ & 0.7610 \\
\hline
\end{tabular}

$73.1 \pm 6.0$ years, the type of corneal astigmatism had already changed to ATR astigmatism at the time of surgery. Accordingly, the increase in ATR corneal astigmatism directly led to an increase in manifest refractive astigmatism. In contrast, because the mean age of eyes having WTR astigmatism was $64.4 \pm 8.1$ years, the type of corneal astigmatism had not yet shifted from WTR astigmatism to ATR astigmatism in most eyes. Thus, because the type of corneal astigmatism was shifting during the study period, the change in manifest refractive astigmatism might not be apparent in eyes having WTR astigmatism. Furthermore, ocular residual astigmatism, which mainly consists of the astigmatism-correcting effect of the toric IOL, did not change significantly over $5 \sim 10$ years postoperatively. Because corneal astigmatism continues to change toward ATR astigmatism over 20 years [30], however, the effect of a toric IOL will deteriorate over time, even in eyes with WTR astigmatism.

Previous studies showed that the astigmatism-correcting effect and rotational stability of toric IOLs are well maintained during a 1- or 2-year follow-up time period 
[14-18]. For example, Miyake et al. [16] reported excellent visual outcomes and rotational stability for up to 2 years postoperatively. No studies to date, however, have evaluated long-term changes in the visual outcomes and refractive effects of toric IOLs for 5 or more years after stabilization of surgically induced astigmatic changes. The present study revealed that the effects of the toric IOL for correcting manifest refractive astigmatism significantly deteriorated with age due to the ATR change in corneal astigmatism, specifically in eyes with ATR astigmatism before surgery.

The present study had several limitations. First, because this study was conducted in a retrospective manner, some of the patient characteristics, including age and sex, differed significantly between the ATR and WTR groups. Corneal astigmatism changes from WTR astigmatism to ATR astigmatism with age [28-31, 33], and this ATR change occurs faster in men than in women [31]; therefore, the distribution of WTR and ATR astigmatism basically differs depending on age and sex. Accordingly, it was difficult to match the age and sex of eyes that underwent implantation of a toric IOL between the ATR and WTR astigmatism groups. Second, because the follow-up duration was only $5 \sim 10$ years, no significant change in uncorrected and corrected distance visual acuity was detected. Changes in the visual outcomes after toric IOL implantation should be examined over a longer time period. Third, we did not examine the long-term refractive changes in eyes having oblique astigmatism. Because only a small number of eyes had the necessary degree of oblique astigmatism for toric IOL implantation during the study period, we did not implant a toric IOL in enough eyes having oblique astigmatism for analysis. Further studies are necessary to evaluate the long-term refractive effect of a toric IOL in eyes having oblique astigmatism.

In conclusion, the refractive effect of the toric IOL significantly deteriorated over time in association with an ATR change of corneal astigmatism in eyes with ATR astigmatism, while it was maintained in eyes with WTR astigmatism. Because the effect of a toric IOL deteriorates with age, overcorrection of ATR astigmatism should be considered depending on the age and sex of the patients scheduled for toric IOL implantation. Further studies are needed to investigate the effects of the toric IOL on visual outcomes for at least 10 years postoperatively.

Supplementary Information The online version contains supplementary material available at https://doi.org/10.1007/s00417-021-05406-7.

Author contribution K.H. and S.H. are responsible for the study conception and design; M.Y. and A.H. are responsible for data acquisition, K.H. is responsible for statistical analysis and interpretation of the data; K.H. is responsible for drafting the manuscript; M.Y., S.H., and A.H. are responsible for reviewing the manuscript; K.H., M.Y., S.H., and
A.H. did the final approval of the manuscript and agreed to be accountable for all aspects of the work in ensuring that questions related to the accuracy or integrity of any part of the work are appropriately investigated and resolved.

Data availability All data that support the findings of this study are available on request from the corresponding author.

\section{Declarations}

Ethical approval The study adhered to the tenets of the Declaration of Helsinki. The Institutional Review Board of the Hayashi Eye Hospital approved the study protocol.

Consent to participate Not applicable.

Consent to publication Not applicable.

Conflict of interest The authors declare no competing interests.

Open Access This article is licensed under a Creative Commons Attribution 4.0 International License, which permits use, sharing, adaptation, distribution and reproduction in any medium or format, as long as you give appropriate credit to the original author(s) and the source, provide a link to the Creative Commons licence, and indicate if changes were made. The images or other third party material in this article are included in the article's Creative Commons licence, unless indicated otherwise in a credit line to the material. If material is not included in the article's Creative Commons licence and your intended use is not permitted by statutory regulation or exceeds the permitted use, you will need to obtain permission directly from the copyright holder. To view a copy of this licence, visit http://creativecommons.org/licenses/by/4.0/.

\section{References}

1. Goggin M, Moore S, Esterman A (2011) Toric intraocular lens outcome using the manufacturer's prediction of corneal plane equivalent intraocular lens cylinder power. Arch Ophthalmol 129:1004-1008. https://doi.org/10.1001/archophthalmol.2011.178

2. Visser N, Beckers HJ, Bauer NJ, Gast ST, Zijlmans BL, Berenschot TT, Webers CA, Nuijts RM (2014) Toric vs aspherical control intraocular lenses in patients with cataract and corneal astigmatism: a randomized clinical trial. JAMA Ophthalmol 132:1462-1468. https://doi.org/10.1001/jamaophthalmol.2014. 3602

3. Krall EM, Arlt EM, Hohensinn M, Moussa S, Jell G, Alió JL, Plaza-Puche AB, Bascaran L, Mendicute J, Grabner G, Dexl AK (2015) Vector analysis of astigmatism correction after toric intraocular lens implantation. J Cataract Refract Surg 41:790-799. https://doi.org/10.1016/j.jcrs.2014.07.038

4. Waltz KL, Featherstone K, Tsai L, Trentacost D (2015) Clinical outcomes of TECNIS toric intraocular lens implantation after cataract removal in patients with corneal astigmatism. Ophthalmology 122:39-47. https://doi.org/10.1016/j.ophtha.2014.06.027

5. Nanavaty MA, Bedi KK, Ali S, Holmes M, Rajak S (2017) Toric intraocular lenses versus peripheral corneal relaxing incisions for astigmatism between 0.75 and 2.5 diopters during cataract surgery. Am J Ophthalmol 180:165-177. https://doi.org/10.1016/j. ajo.2017.06.007

6. Mayer WJ, Kreutzer T, Dirisamer M, Kern C, Kortuem K, Vounotrypidis E, Priglinger S, Kook D (2017) Comparison of 
visual outcomes, alignment accuracy, and surgical time between 2 methods of corneal marking for toric intraocular lens implantation. J Cataract Refract Surg 43:1281-1286. https://doi.org/10. 1016/j.jcrs.2017.07.030

7. Webers VSC, Bauer NJC, Visser N, Berendschot TTJM, van den Biggelaar FJHM, Nuijts RMMA (2017) Image-guided system versus manual marking for toric intraocular lens alignment in cataract surgery. J Cataract Refract Surg 43:781-788. https://doi.org/10. 1016/j.jcrs.2017.03.041

8. Solomon KD, Sandoval HP, Potvin R (2019) Evaluating the relative value of intraoperative aberrometry versus current formulas for toric IOL sphere, cylinder, and orientation planning. J Cataract Refract Surg 45:1430-1435. https://doi.org/10.1016/j.jcrs.2019. 05.023

9. Zhou F, Jiang W, Lin Z, Li X, Li J, Lin H, Chen W, Wang Q (2019) Comparative meta-analysis of toric intraocular lens alignment accuracy in cataract patients: image-guided system versus manual marking. J Cataract Refract Surg 45:1340-1345. https:// doi.org/10.1016/j.jcrs.2019.03.030

10. Gangwani V, Hirnschall N, Findl O, Maurino V (2014) Multifocal toric intraocular lenses versus multifocal intraocular lenses combined with peripheral corneal relaxing incisions to correct moderate astigmatism. J Cataract Refract Surg 40:1625-1632. https://doi.org/10.1016/j.jcrs.2014.01.037

11. Kretz FT, Bastelica A, Carreras H, Ferreira T, Müller M, Gerl M, Gerl R, Saeed M, Schmickler S, Auffarth GU (2015) Clinical outcomes and surgeon assessment after implantation of a new diffractive multifocal toric intraocular lens. Br J Ophthalmol 99:405-411. https://doi.org/10.1136/bjophthalmol-2014-305570

12. Kretz FT, Breyer D, Klabe K, Hagen P, Kaymak H, Koss MJ, Gerl M, Mueller M, Gerl RH, Auffarth GU (2015) Clinical outcomes after implantation of a trifocal toric intraocular lens. J Refract Surg 31:504-510. https://doi.org/10.3928/1081597X-20150 622-01

13. Hayashi K, Masumoto M, Takimoto M (2015) Comparison of visual and refractive outcomes after bilateral implantation of toric intraocular lenses with or without a multifocal component. J Cataract Refract Surg 41:73-83. https://doi.org/10.1016/j.jcrs.2014.04. 032

14. Kwartz J, Edwards K (2010) Evaluation of the long-term rotational stability of single-piece, acrylic intraocular lenses. Br J Ophthalmol 94:1003-1006. https://doi.org/10.1136/bjo.2009. 163485

15. Kim MH, Chung TY, Chung ES (2010) Long-term efficacy and rotational stability of AcrySof toric intraocular lens implantation in cataract surgery. Korean J Ophthalmol 24:207-212. https://doi. org/10.3341/kjo.2010.24.4.207

16. Miyake T, Kamiya K, Amano R, Iida Y, Tsunehiro S, Shimizu K (2014) Long-term clinical outcomes of toric intraocular lens implantation in cataract cases with preexisting astigmatism. J Cataract Refract Surg 40:1654-1660. https://doi.org/10.1016/j. jcrs.2014.01.044

17. Gyöngyössy B, Jirak P, Schönherr U (2017) Long-term rotational stability and visual outcomes of a single-piece hydrophilic acrylic toric IOL: a 1.5-year follow-up. Int J Ophthalmol 10:573-578. https://doi.org/10.18240/ijo.2017.04.12

18. Fernández-Muñoz E, Blanco-Gómez J, Vázquez-Rojas R, Camargo-Ibarias K, Massaro-Corredor M, Gonzalez-Salinas R (2020) Long-term refractive outcomes in patients with cataracts and keratoconus after phacoemulsification with toric intraocular lens implant. Eur J Ophthalmol. https://doi.org/10.1177/1120672120 960585

19. Koch DD, Ali SF, Weikert MP, Shirayama M, Jenkins R, Wang L (2012) Contribution of posterior corneal astigmatism to total corneal astigmatism. J Cataract Refract Surg 38:2080-2087. https:// doi.org/10.1016/j.jcrs.2012.08.036
20. Koch DD, Jenkins RB, Weikert MP, Yeu E, Wang L (2013) Correcting astigmatism with toric intraocular lenses: effect of posterior corneal astigmatism. J Cataract Refract Surg 39:1803-1809. https://doi.org/10.1016/j.jcrs.2013.06.027

21. Reitblat O, Levy A, Kleinmann G, Abulafia A, Assia EI (2016) Effect of posterior corneal astigmatism on power calculation and alignment of toric intraocular lenses: comparison of methodologies. J Cataract Refract Surg 42:217-225. https://doi.org/10. 1016/j.jcrs.2015.11.036

22. Ninomiya Y, Minami K, Miyata K, Eguchi S, Sato R, Okamoto F, Oshika T (2016) Toric intraocular lenses in eyes with with-therule, against-the-rule, and oblique astigmatism: one-year results. J Cataract Refract Surg 42:1431-1440. https://doi.org/10.1016/j. jcrs.2016.07.034

23. Ferreira TB, Ribeiro P, Ribeiro FJ, O'Neill JG (2017) Comparison of methodologies using estimated or measured values of total corneal astigmatism for toric intraocular lens power calculation. J Refract Surg 33:794-800. https://doi.org/10.3928/1081597X20171004-03

24. Alpins NA (1997) New method of targeting vectors to treat astigmatism. J Cataract Refract Surg 23:65-75. https://doi.org/10. 1016/s0886-3350(97)80153-8

25. Kansara N, Cui D, Banerjee K, Landis Z, Scott IU, Pantanelli SM (2021) Anterior, posterior, and non-keratometric contributions to refractive astigmatism in pseudophakes. J Cataract Refract Surg 47:93-99. https://doi.org/10.1097/j.jcrs.0000000000000390

26. Naeser K, Hjortdal J (2001) Multivariate analysis of refractive data: mathematics and statistics of spherocylinders. J Cataract Refract Surg 27:129-142. https://doi.org/10.1016/s0886-3350(00) 00816-6

27. Abulafia A, Koch DD, Holladay JT, Wang L, Hill W (2018) Pursuing perfection in intraocular lens calculations: IV. Rethinking astigmatism analysis for intraocular lens-based surgery: Suggested terminology, analysis, and standards for outcome reports. J Cataract Refract Surg 44:1169-1174. https://doi.org/10.1016/j.jcrs. 2018.07.027

28. Hayashi K, Hirata A, Manabe S, Hayashi H (2011) Long-term change in corneal astigmatism after sutureless cataract surgery. Am J Ophthalmol 151:858-865. https://doi.org/10.1016/j.ajo. 2010.11.014

29. Hayashi K, Ogawa S, Manabe S, Hirata A (2015) Influence of patient age at surgery on long-term corneal astigmatic change subsequent to cataract surgery. Am J Ophthalmol 160:171-178. https://doi.org/10.1016/j.ajo.2015.04.015

30. Hayashi K, Manabe SI, Hirata A, Yoshimura K (2017) Changes in corneal astigmatism during 20 years after cataract surgery. J Cataract Refract Surg 43:615-621. https://doi.org/10.1016/j.jcrs. 2017.02.028

31. Hayashi K, Sato T, Sasaki H, Hirata A, Yoshimura K (2018) Sexrelated differences in corneal astigmatism and shape with age. J Cataract Refract Surg 44:1130-1139. https://doi.org/10.1016/j. jcrs.2018.06.020

32. Hayashi K, Yoshida M, Hayashi S, Yoshimura K. Long-term changes in manifest refraction subsequent to cataract surgery. J Cataract Refract Surg in press

33. Hayashi K, Hayashi H, Hayashi F (1995) Topographic analysis of the changes in corneal shape due to aging. Cornea 14:527-532

Publisher's note Springer Nature remains neutral with regard to jurisdictional claims in published maps and institutional affiliations. 


\section{Authors and Affiliations}

\section{Ken Hayashi $^{1}$ - Motoaki Yoshida ${ }^{1} \cdot$ Shunsuke Hayashi $^{2,3} \cdot$ Akira Hirata $^{1}$}

1 Hayashi Eye Hospital, 4-7-13 Hakataekimae, Hakata-Ku, Fukuoka 812-0011, Japan

2 Department of Ophthalmology, National Hospital Organization of Saitama Hospital, Wako, Japan
3 Department of Ophthalmology, Faculty of Medicine, Keio University, Tokyo, Japan 